\title{
Sebaceous glands in the esophagus-new approach to metaplastic theory: a case report and review of the literature
}

\author{
Vuka Katic $^{1,2 *}$, Zoran Radovanovic ${ }^{1}$, Bratislav Petrovic ${ }^{1}$, Vesna Brzacki ${ }^{1}$, Dejan Popovic ${ }^{1}$ and Sasa Grgov ${ }^{1}$ \\ ${ }^{1}$ University of Nis, Sumatovacka, Nis, Serbia \\ ${ }^{2}$ Department of Pathology in Human Policlinic, University of Nis, Sumatovacka, Nis, Serbia
}

\begin{abstract}
Ectopic salivary glands in the esophagus have rarely been reported, representing an obscure medical condition. The endoscopic findings of the EESg indicated multiple yellowish patches or papules, which varied in size. The histopathological findings indicated several lobulated sebaceous glands in the squamous epithelium with inflammatory infiltration. Esophageal "ectopic" sebaceous glands do not require further treatment or follow-up.
\end{abstract}

\section{Background}

Metaplasia is the conversion of one differentiate cell type to another [1]. Metaplastic lesions have been recognized in many tissues of endodermal origin, especialy in the stomach and in the Barrett esophagitis [2,3]. In contrast, heterotopic sebaceous glands (HSGs) in the esophagus are considered to be very rare, representing an obscure medical condition [4-8]. Some authors have tried to determine whether HSGs is result of a metaplastic process or a heterotopic congenital anomaly? However, the answer is not yet known [6-8]. Often localisation of HSGs is in the middle and lower esophagus in numbers ranging from 1 to more than 100 yellowish plaques, measuring 1-2 mm in diameter, often without any clinical symptom [8-10].

\section{Aim}

Having in the mind the pointed-out dilemma on the pathogenesis of this rare esophageal process, we have undertaken the following study: the identification of both clinical and patohistological features in the patient with rare esophageal sebaceous process.

\section{Case report}

A 46 year-old-woman, presented with a history of discomfort during swallowing, since the last 6 months. There was no history of vomiting, hematemesis, or weight loss. The patient was not a chronic smoker. General physical examination was unremarkable. Respiratory and cardiovascular systems were normal. Symptomatic treatment, with proton pump inhibitors, has been given. No significant abnormality was found on physical examination, or laboratory tests. Clinically, carcinoma of the esophagus was suspected and the esophagogastrointestinal endoscopy has been done. Seven wart-like yellowish papillomatous projections which varied in size, in the lower esophagus, were observed.

Pinch biopsy from one, of seven projections, was taken and sent for histological examination. Paraffin biopsy blocks were cut and stained with HE and PAS methods. Microscopically, the squamous epithelial lining was fragmented, showing hiperkerathosis (Figure 1).

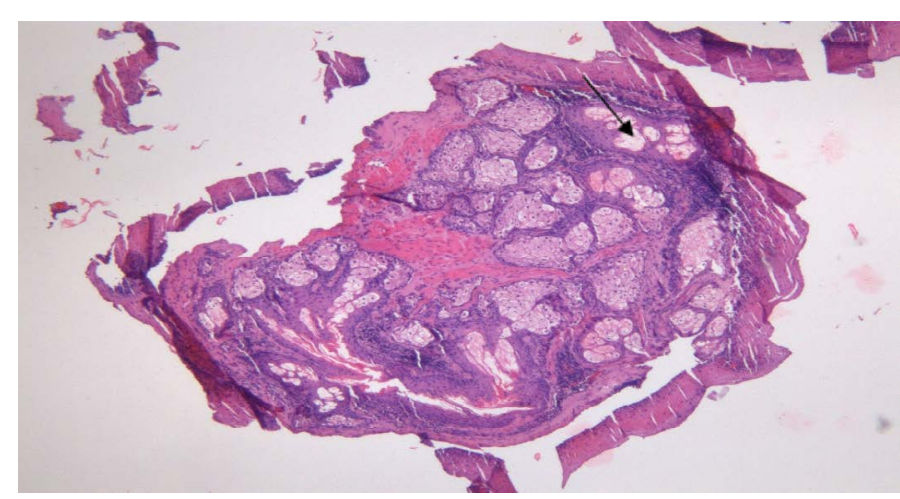

Figure 1. Esophageal squamous epithelium with sebaceous glands in the propria. HE x 200 .

The presence of the esophageal mucinous glands of white color, in the subepithelial area, was revealed (two arrows).

The mostly of subepithelial space was filled with metaplastic sebaceous glands: they were lobulated. with polygonal cells, that have small nuclei and abundant clear cytoplasm, showing microvesicular vacuolisation and typical sebaceous differentiation (Figure 2). Between these cells, could be seen the remnants of the mucinous cells of small size, confirming the metaplastic theory De La Pava et al. [4]. The second microscopical proof for metaplastic theory is chronic inflammation: dense lymphocytic and macrophage infiltration, surrounded by dense fibrous, sometimes by hyalinized stroma, that provide physical support to multilobular structures [5-8]. Endly, our patient is relatively old woman, not child. On the base of pointed out criterions, histological diagnosis of the sebaceous metaplasia in the esophagus, was made.

${ }^{\star}$ Correspondence to: Vuka Katic, Medical Faculty of Nis, University of Nis, Sumatovacka, Nis, Serbia, Tel: +00381 18 533190; E-mail: vuka.katic@gmail.com

Key words: haematemesis, lower esophagus, squamous epithelial lining

Received: April 01, 2018; Accepted: April 25, 2018; Published: April 29, 2018 


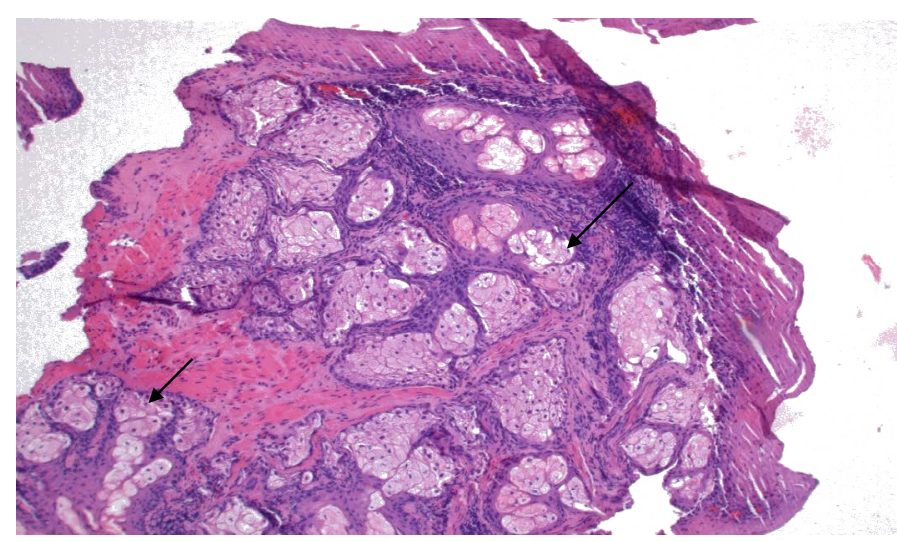

Figure 2. Sebaceous metaplasia: large polygonal clear cells with micro vesicular vacuolization; focal, mild squamous epithelial hyperplasia. HE x 400

The patient was treated symptomatically with antacids and omeprazole for one month, and she became symptoms free and remained so at one year of follow-up. Histogenesis of this rare lesion is discussed in this case report: ESGs are derived from the ectoderm. There was no history of vomiting or hematemesis or, weight loss. HCGs are very rare esophageal conditions with an incidence around $0.00465 \%$ and an occurence rate of 0.41 per year (1-6; 9-110). Despite the extreme rarity at this entity, it is considered a lesion with no malignant potential [7-14].

\section{Discussion}

Many years have passed from the tume (1962) when De La Pava et al. [4] were discovered the ectopic sebaceoud glands in the middle and lower esophagus. The authors reported such occurence in 4 of the 200 unselected cadavers. However, in that time it was first reported presence of SGs (ectodermal) in an endodermally organ. The primary endoscopic impression was papilloma, but microscopical study proved, to be sebaceous glands, but unaccompanied by hair follicles. However, in that time (1962) the same authors first reported the presence of SGs in an endodermal derived organ- esophagus, in a 44- year- old nonsmoker. Histological examination identified numerous sebaceous glands in the lamina propria, showing the characteristics of sebaceous differentiation. Histogenesis of this process is not completely clear. To explain the presence of ectodermal tissue (sebaceous gland) in the endodermal orgasm (esophagus), several hypotheses have been postulated [5-9].

Metaplastic theory: this is favored by the occurrence of this condition in the elderly, not in children, and by the arrangement of these glands which is like that of esophageal submucous glands. In addition, chronic inflammation with lymphocytic and macrophagic cells must be also present.

Heterotopic theory: This thesis is not supported by endoscopic studies and autopsy studies conducted on infants or children [10]. HEGs in esophagus are a very rare occurrence. It probably represents a metaplastic change in the esophageal submucosal glands. Here, we confirm this metaplastic sebaceous rare occurrence in a 45 year-old female, by the following characteristics. Histogenesis is not completely clear. Several hypotheses have been postulated: metaplasia heterotopia.
Metaplasia theory: The occurrence of this condition only in elderly is the reason for more frequent its accepting in the science. We discovered inside sebaceous lobules in the lamina propria the rest of normal mucinous cells, confirms metaplastic theory. In addition, rare finding the sebaceous metaplasia in esophageal mucosa can be explained great risk of gastroenterologists for taking submucosal biopsy because esophageal venous varices localized in the submucosal layer of the esophagus, in lower third of esophagus, the same location sebaceous glands and varices in the esophageal mucosa. Heterotopia theory (embryological misplacement) This theory is not supported by endoscopic studies and autopsy studies conducted on infants and children

\section{Conclusions}

- Sebaceous glands in the esophagus are very rare.

- Clinical symptoms, sex and endoscopic finding are not specific.

- Tiny yellowish lesions, of submucous localization in the lamina propria of the lower esophagus, are not specific.

- The most important discover is microscopically: normal, small, mucinous submucous esophageal glands and their multilobular sebaceous metaplasia in "sebaceous" glands.

\section{References}

1. Filipe MI, Jass JR (1986) Intestinal metaplasia subtypes and cancer risk. Churchil Livingstone, London, pp: 87-115.

2. Zak FG, Lawson W (1976) Sebaceous glands in the esophagus. First case observed grossly. Arch Dermatol 112: 1153-1154. [Crossref]

3. Macukanovic-Golubovic L, Katic V, Rancic G, Milenovic M, Golubovic Z (2007) Study on histogenesis of Enterochromaffine-like carcinoid in autoimmune gastritis associated with pernicious anaemia. Vojnosanit Pregl 64: 507-580.

4. http://openaccessebooks.com/digestive-system-anatomy/study-on-morphology-andpathogenesis-of-type-1-ECL-cell-gastric-carcinoids.pdf

5. De La Pava S, Pickren JW (1962) Ectopic sebaceous glands in the esophagus. Arch Pathol 73: 397-399.

6. Bahat RV, Ramaswamy RR, Yelagondahally LKC (2008) Ectopic sebaceous glands in the Esophagus: a case report and Review of literature. Saudi J Gastroenterol 14 83-84. [Crossref]

7. Jhang LS, Hung CY, Chien IY, Chen MJ (2016) Ectopic sebaceous glands in the esophagus-Case reports and review of the literature. Adv Dig Med 3: 118-122.

8. Chiu KW, Wu CK, Lu LS, Eng HL, Chiou SS (2014) Diagnostic pitfall of sebaceous gland metaplasia of the esophagus. World J Clin cases 2: 311-315. [Crossref]

9. Nakada T, Inoue F, Iwasaki M (1995) Ectopic Sebaceous glands in the esophagus. $\mathrm{Am}$ J Gastroenterol 90: 501-503.

10. Nishisaki H, Yasutake K, Makashima T, Hasegawa H (2007) Five cases with ectopic Esophageal sebaceous glands. Dig Endosc 9: 207-212.

11. Park A, Lee JH, Park A, Jung YH, Chu HJ, et al. (2017) Prevalence rate and clinic characteristics of ESEGs in asymptomatic health screen of examinees. Dis Esophagus 30: $1-5$. [Crossref]

12. Montalvo N, Tapia V, Padilla H, Redrobán L (2017) Heterotopic sebaceous glands in the esophagus, a very rare histopathological diagnosis: a case report and review of the literature. Clin Case Rep 5: 89-92. [Crossref]

13. Bae JY, Chon CY, Kim H (1996) Sebaceous glands in the esophagus. J Korean Med Sci 11: 271-274.

14. Thalheimer U, Wright JL, Maxwell P, Firth J, Millar A (2008) Sebaceous glands in the Esophagus. Endoscopy 40: E57.

Copyright: (C2018 Katic V. This is an open-access article distributed under the terms of the Creative Commons Attribution License, which permits unrestricted use, distribution, and reproduction in any medium, provided the original author and source are credited. 